\title{
Local convergence of Newton's method for solving generalized equations with monotone operator
}

\author{
Gilson N. Silva *
}

July 29, 2016

\begin{abstract}
In this paper we study Newton's method for solving the generalized equation $F(x)+T(x) \ni 0$ in Hilbert spaces, where $F$ is a Fréchet differentiable function and $T$ is set-valued and maximal monotone. We show that this method is local quadratically convergent to a solution. Using the idea of majorant condition on the nonlinear function which is associated to the generalized equation, the convergence of the method, the optimal convergence radius and results on the convergence rate are established. The advantage of working with a majorant condition rests in the fact that it allow to unify several convergence results pertaining to Newton's method.
\end{abstract}

Keywords: Generalized equation, Newton's method, Majorant condition, Banach lemma.

\section{Introduction}

The idea of solving a generalized equation of the form

$$
\text { Find } x \text { such that } F(x)+T(x) \ni 0 \text {, }
$$

where $F: \Omega \rightarrow H$ is a Fréchet differentiable function, $H$ is a Hibert space, $\Omega \subseteq H$ an open set and $T: H \rightrightarrows H$ is a set-valued and maximal monotone, plays a huge role in classical analysis and its applications. For instance, systems of nonlinear equations and abstract inequality systems. If $\psi: H \rightarrow(-\infty,+\infty]$ is a proper lower semicontinuous convex function and

$$
T(x)=\partial \psi(x)=\{u \in H \quad: \psi(y) \geq \psi(x)+\langle u, y-x\rangle\}, \quad \forall \quad y \in H,
$$

then (11) becomes the variational inequality problem

$$
F(x)+\partial \psi(x) \ni 0
$$

including linear and nonlinear complementary problems; additional comments about such problems can be found in [5, 7, $8,11,14,17,20,22$.

Newton's method has been extended in order to solve nonlinear systems of equalities and inequalities (see [3] ). In particular, Robinson in [17] generalized Newton's method for solving problems of the form

$$
F(x) \in C,
$$

${ }^{*}$ CCET/UFOB, CEP 47808-021 - Barreiras, BA, Brazil (Email: gilson.silva@ufob.edu.br). 
which becomes the usual Newton's method to the special case in which $C$ is the degenerate cone $\{0\} \subset Y$.

A Newton method for solving (11) utilizes the iteration

$$
F\left(x_{k}\right)+F^{\prime}\left(x_{k}\right)\left(x_{k+1}-x_{k}\right)+F\left(x_{k+1}\right) \ni 0, k=0,1, \ldots
$$

for $x_{0}$ a given initial point. As is well known, the generalized equation (1) covers huge territory in classical analysis and its applications. When $F \equiv 0$, the iteration (2) becomes the standard Newton method for solving the nonlinear equation $F(x)=0$,

$$
F\left(x_{k}\right)+F^{\prime}\left(x_{k}\right)\left(x_{k+1}-x_{k}\right)=0, \quad k=0,1, \ldots
$$

In [2, 21] under a majorant condition and generalized Lipschitz condition, local and semi local convergence, quadratic rate and estimate of the best possible convergence radius of Newton's method as well as uniqueness of the solution for solving generalized equation were established.

It is well-known that an assumption used to obtain quadratic convergence of Newton's method (2), for solving equation (11), is the Lipschitz continuity of $F^{\prime}$ in a neighborhood of the solution. Indeed, keeping control of the derivative is an important point in the convergence analysis of Newton's method. On the other hand, a couple of papers have dealt with the issue of convergence analysis of the Newton's method, for solving the equation $F(x)=0$, by relaxing the assumption of Lipschitz continuity of $F^{\prime}$, see for example [12, 13, 23, 24]. The advantage of working with a majorant condition rests in the fact that it allow to unify several convergence results pertaining to Newton's method; see [12, 23. In this paper we work with the majorant condition introduced in [12. The analysis presented provides a clear relationship between the majorant function and the function defining the generalized equation. Also, it allows us to obtain the optimal convergence radius for the method with respect to the majorant condition and uniqueness of solution. The analysis of this method, under Lipschitz's condition and Smale's condition, are provided as special case.

The organization of the paper is as follows. In Section 2, some notations and important results used throughout the paper are presented. In Section 3. the main result is stated and in Section 3.1 properties of the majorant function, the main relationships between the majorant function and the nonlinear operator, the uniqueness of the solution and the optimal convergence radius are established. In Section 3.2, the main result is proved and in the last section some applications of this result are given.

\section{Preliminaries}

The following notations and results are used throughout our presentation. Let $H$ be a Hilbert space with scalar product $\langle.,$.$\rangle and norm \|$.$\| , the open and closed balls at x$ with radius $\delta \geq 0$ are denoted, respectively, by $B(x, \delta)$ and $B[x, \delta]$.

We denote by $\mathscr{L}(X, Y)$ the space consisting of all continuous linear mappings $A: X \rightarrow Y$ and the operator norm of $A$ is defined by $\|A\|:=\sup \{\|A x\|:\|x\| \leqslant 1\}$. A bounded linear operator $G: H \rightarrow H$ is called a positive operator if $G$ is a self-conjugate and $\langle G x, x\rangle \geq 0$ for each $x \in H$. The domain and the range of $G$ are, respectively, the sets $\operatorname{dom} G:=\{x \in H: G(x) \neq \varnothing\}$ and rge $G:=\{y \in H: y \in G(x)$ for some $x \in X\}$. The inverse of $G$ is the set-valued mapping $G^{-1}: H \rightrightarrows H$ defined by $G^{-1}(y):=\{x \in H: y \in G(x)\}$.

Now, we recall notions of monotonicity for set-valued operators. 
Definition 1. Let $T: H \rightrightarrows H$ be a set-valued operator. $T$ is said to be monotone if for any $x, y \in \operatorname{dom} T$ and, $u \in T(y), v \in T(x)$ implies that the following inequality holds:

$$
\langle u-v, y-x\rangle \geq 0 \text {. }
$$

A subset of $H \times H$ is monotone if it is the graph of a monotone operator. If $\varphi: H \rightarrow(-\infty,+\infty]$ is a proper function then the subgradient of $\varphi$ is monotone.

Definition 2. Let $T: H \rightrightarrows H$ be monotone. Then $T$ is maximal monotone if the following implication holds for all $x, u \in H$ :

$\langle u-v, y-x\rangle \geq 0 \quad$ for each $y \in \operatorname{dom} T$ and $\quad v \in T(y) \Rightarrow \quad x \in \operatorname{dom} T$ and $\quad v \in T(x)$.

An example of maximal monotone operator is the subdifferential of a proper, lower semicontinuous, convex function $\varphi: H \rightarrow(-\infty,+\infty]$. The following result can de found in [22].

Lemma 1. Let $G$ be a positive operator. The following statements about $G$ hold:

1. $\left\|G^{2}\right\|=\|G\|^{2}$;

2. If $G^{-1}$ exists, then $G^{-1}$ is a positive operator.

As a consequence of this result we have the following result:

Lemma 2. Let $G$ be a positive operator. Suppose that $G^{-1}$ exists, then for each $x \in H$ we have

$$
\langle G x, x\rangle \geq \frac{\|x\|^{2}}{\left\|G^{-1}\right\|} .
$$

Proof. See Lemma 2.2 of [20].

Let $G: H \rightarrow H$ be a bounded linear operator. We will use the convention that $\widehat{G}:=\frac{1}{2}\left(G+G^{*}\right)$ where $G^{*}$ is the conjugate operator of $G$. As we can see, $\widehat{G}$ is a self-conjugate operator. From now, we assume that $T: H \rightrightarrows H$ is a set-valued maximal monotone operator and $F: H \rightarrow H$ is a Fréchet derivative function. The next result is of major importance to prove the good definition of Newton's method. Its proof can be found in [18, Lemma 1, p.189].

Lemma 3 (Banach's lemma). Let $B: H \rightarrow H$ be a bounded linear operator and $I: H \rightarrow H$ the identity operator. If $\|B-I\|<1$ then $B$ is invertible and $\left\|B^{-1}\right\| \leq 1 /(1-\|B-I\|)$.

\section{Local analysis of Newton's method}

In this section, we study the Newton's method for solving (1). For study the convergence properties of this method, we assume that the derivative $F^{\prime}$ satisfies a weak Lipschitz condition on a region $\Omega$ relaxing the usual Lipschitz condition. The statement of the our main result is:

Theorem 4. Let $H$ be a Hilbert space, $\Omega$ be an open nonempty subset of $H, F: \Omega \rightarrow H$ be continuous with Fréchet derivative $F^{\prime}$ continuous, $T: H \rightrightarrows H$ be a set-valued operator and $x^{*} \in \Omega$. Suppose that $0 \in F\left(x^{*}\right)+T\left(x^{*}\right), F^{\prime}\left(x^{*}\right)$ is a positive operator and ${\widehat{F^{\prime}\left(x^{*}\right)}}^{-1}$ exists. Let $R>0$ and $\kappa:=\sup \left\{t \in[0, R): B\left(x^{*}, t\right) \subset \Omega\right\}$. Suppose that there exists $f:[0, R) \rightarrow \mathbb{R}$ twice continuously differentiable such that

$$
\left\|{\widehat{F^{\prime}\left(x^{*}\right)}}^{-1}\right\|\left\|F^{\prime}(x)-F^{\prime}\left(x^{*}+\tau\left(x-x^{*}\right)\right)\right\| \leq f^{\prime}\left(\left\|x-x^{*}\right\|\right)-f^{\prime}\left(\tau\left\|x-x^{*}\right\|\right),
$$

for all $\tau \in[0,1], x \in B\left(x^{*}, \kappa\right)$ and 
h1) $f(0)=0$ and $f^{\prime}(0)=-1$;

h2) $f^{\prime}$ is convex and strictly increasing.

Let $\nu:=\sup \left\{t \in[0, R): f^{\prime}(t)<0\right\}, \rho:=\sup \left\{t \in(0, \nu): f(t) /\left(t f^{\prime}(t)\right)-1<1\right\}$ and $r:=\min \{\kappa, \rho\}$. Then, the sequences with starting point $x_{0} \in B\left(x^{*}, r\right) /\left\{x^{*}\right\}$ and $t_{0}=\left\|x^{*}-x_{0}\right\|$, respectively,

$$
0 \in F\left(x_{k}\right)+F^{\prime}\left(x_{k}\right)\left(x_{k+1}-x_{k}\right)+T\left(x_{k+1}\right), \quad t_{k+1}=\left|t_{k}-f\left(t_{k}\right) / f^{\prime}\left(t_{k}\right)\right|, \quad k=0,1, \ldots,
$$

are well defined, $\left\{t_{k}\right\}$ is strictly decreasing, is contained in $(0, r)$ and converges to $0,\left\{x_{k}\right\}$ is contained in $B\left(x^{*}, r\right)$ and converges to the point $x^{*}$ which is the unique solution of the generalized equation $F(x)+T(x) \ni 0$ in $B\left(x^{*}, \bar{\sigma}\right)$, where $\bar{\sigma}=\min \{r, \sigma\}$ and $\sigma:=\sup \{0<t<\kappa: f(t)<0\}$. Moreover, the sequence $\left\{t_{k+1} / t_{k}^{2}\right\}$ is strictly decreasing,

$$
\left\|x^{*}-x_{k+1}\right\| \leq\left[t_{k+1} / t_{k}^{2}\right]\left\|x_{k}-x^{*}\right\|^{2}, \quad t_{k+1} / t_{k}^{2} \leq f^{\prime \prime}\left(t_{0}\right) /\left(2\left|f^{\prime}\left(t_{0}\right)\right|\right), \quad k=0,1, \ldots .
$$

If, additionally, $f(\rho) /\left(\rho f^{\prime}(\rho)\right)-1=1$ and $\rho<\kappa$, then $r=\rho$ is the optimal convergence radius.

Remark 1. Combining inequalities in (7), we obtain that $\left\{x_{k}\right\}$ converges Q-quadratically to $\bar{x}$. Moreover, as $\left\{t_{k+1} / t_{k}^{2}\right\}$ is strictly decreasing we have $t_{k+1} / t_{k}^{2}<t_{1} / t_{0}^{2}$, for $k=0,1, \ldots$ Thus, first inequality in (17) implies $\left\|\bar{x}-x_{k+1}\right\| \leq\left[t_{1} / t_{0}^{2}\right]\left\|x_{k}-\bar{x}\right\|^{2}$, for $k=0,1, \ldots$ As a consequence,

$$
\left\|\bar{x}-x_{k}\right\| \leq t_{0}\left(t_{1} / t_{0}\right)^{2^{k}-1}, \quad k=0,1, \ldots
$$

Remark 2. Since $T$ is monotone maximal, if there exists a constant $c>0$ such that

$$
\left\langle F^{\prime}\left(x_{k}\right) y, y\right\rangle \geq c\|y\|^{2}
$$

for each $y \in H$, then there exists a unique point $x_{k+1}$ such that the first inclusion in (6) holds. The proof of this result can be found in [20, Lemma 2.2]. Hence, if for each $k$, there exists a constant $c>0$ such that (8) holds, then the sequence generated by (6) is well defined.

From now on, we assume that the hypotheses of Theorem 4 hold.

\subsection{Basic results}

In this section, we establish some relationships between the majorant function $f$ and the set-valued mapping $F+T$. The Proposition 2.5 of [9] state that the constants $\kappa, \nu$ and $\sigma$ are all positive and $t-f(t) / f^{\prime}(t)<0$, for all $t \in(0, \nu)$. According to $\mathbf{h} \mathbf{2}$ and definition of $\nu$, we have $f^{\prime}(t)<0$ for all $t \in[0, \nu)$. Therefore, the Newton iteration map for $f$ is well defined in $[0, \nu)$, namely, $n_{f}:[0, \nu) \rightarrow(-\infty, 0]$ is defined by

$$
n_{f}(t):=t-f(t) / f^{\prime}(t), \quad t \in[0, \nu) .
$$

The next proposition was proved in Proposition 2.6 and Proposition 2.7 of [9].

Proposition 5. The mapping $(0, \nu) \ni t \mapsto\left|n_{f}(t)\right| / t^{2}$ is strictly increasing and

$$
\left|n_{f}(t)\right| / t^{2} \leq f^{\prime \prime}(t) /\left(2\left|f^{\prime}(t)\right|\right),
$$

for all $t \in(0, \nu)$. Moreover, the constant $\rho$ is positive. As a consequence, $\left|n_{f}(t)\right|<t$ for all $t \in(0, \rho)$. 
Using (9), it is easy to see that the sequence $\left\{t_{k}\right\}$ is equivalently defined as

$$
t_{0}=\left\|x^{*}-x_{0}\right\|, \quad t_{k+1}=\left|n_{f}\left(t_{k}\right)\right|, \quad k=0,1, \ldots .
$$

Next result contain the main convergence properties of the above sequence and its prove is similar to Corollary 2.8 of $[9]$.

Corollary 6. The sequence $\left\{t_{k}\right\}$ is well defined, is strictly decreasing and is contained in $(0, \rho)$. Moreover, $\left\{t_{k+1} / t_{k}^{2}\right\}$ is strictly decreasing, $\left\{t_{k}\right\}$ converges to 0 and $t_{k+1} / t_{k}^{2} \leq\left[f^{\prime \prime}\left(t_{0}\right) /\left(2\left|f^{\prime}\left(t_{0}\right)\right|\right)\right]$, for $k=0,1, \ldots$

In the sequel we will prove that the partial linearization of $F+T$ has a single-valued inverse, which is Lipschitz in a neighborhood of $x^{*}$. Since Newton's iteration at a point in this neighborhood happens to be a zero of the partial linearization of $F+T$ at such a point, it will be first convenient to study the linearization error of $F$ at a point in $\Omega$

$$
E_{F}(x, y):=F(y)-\left[F(x)+F^{\prime}(x)(y-x)\right], \quad y, x \in \Omega .
$$

In the next result we bound this error by the linearization error of the majorant function $f$, namely,

$$
e_{f}(t, u):=f(u)-\left[f(t)+f^{\prime}(t)(u-t)\right], \quad t, u \in[0, R) .
$$

Lemma 7. There holds $\left\|{\widehat{F^{\prime}\left(x^{*}\right)}}^{-1}\right\|\left\|E_{F}\left(x, x^{*}\right)\right\| \leq e_{f}\left(\left\|x^{*}-x\right\|, 0\right)$, for all $x \in B\left(x^{*}, \kappa\right)$.

Proof. Since $x^{*}+(1-u)\left(x-x^{*}\right) \in B\left(x^{*}, \kappa\right)$, for all $0 \leq u \leq 1$ and $F$ is continuously differentiable in $\Omega$, thus the definition of $E_{F}$ and some simple manipulations yield

$$
\left.\left\|{\widehat{F^{\prime}\left(x^{*}\right)}}^{-1}\right\|\left\|E_{F}\left(x, x^{*}\right)\right\| \leq \int_{0}^{1}\left\|{\widehat{F^{\prime}\left(x^{*}\right)}}^{-1}\right\| \| F^{\prime}(x)-F^{\prime}\left(x^{*}+(1-u)\left(x-x^{*}\right)\right)\right]\|\| x^{*}-x \| d u .
$$

Combining last inequality with (5) with $\tau=1-u$ and then performing the integral obtained using that $f(0)=0$ we obtain that

$$
\begin{aligned}
\left\|{\widehat{F^{\prime}\left(x^{*}\right)}}^{-1}\right\|\left\|E_{F}\left(x, x^{*}\right)\right\| & \leq \int_{0}^{1}\left[f^{\prime}\left(\left\|x^{*}-x\right\|\right)-f^{\prime}\left((1-u)\left\|x^{*}-x\right\|\right)\right]\left\|x^{*}-x\right\| d u \\
& =f^{\prime}\left(\left\|x^{*}-x\right\|\right)\left\|x^{*}-x\right\|-f\left(\left\|x^{*}-x\right\|\right) .
\end{aligned}
$$

Therefore using $h_{1}$ and the definition of $e_{f}$ the statement follows.

In the next result we will present the main relationships between the majorant function $f$ and the operator $F$. The result is a consequence of Banach's lemma and its statement is:

Lemma 8. Let $x^{*} \in H$ be such that $\widehat{F^{\prime}\left(x^{*}\right)}$ is a positive operator and ${\widehat{F^{\prime}\left(x^{*}\right)}}^{-1}$ exists. If $\left\|x-x^{*}\right\| \leq$ $\min \{\kappa, \nu\}$, then $\widehat{F^{\prime}(x)}$ is a positive operator and ${\widehat{F^{\prime}(x)}}^{-1}$ exists. Moreover,

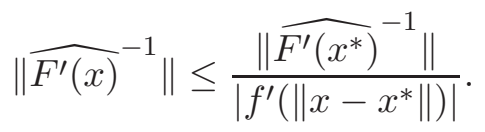


Proof. Firstly note that

$$
\left\|\widehat{F^{\prime}(x)}-\widehat{F^{\prime}\left(x^{*}\right)}\right\| \leq \frac{1}{2}\left\|F^{\prime}(x)-F^{\prime}\left(x^{*}\right)\right\|+\frac{1}{2}\left\|\left(F^{\prime}(x)-F^{\prime}\left(x^{*}\right)\right)^{*}\right\|=\left\|F^{\prime}(x)-F^{\prime}\left(x^{*}\right)\right\| .
$$

Take $x \in B\left(x^{*}, r\right)$. Since $r<\nu$ we have $\left\|x-x^{*}\right\|<\nu$. Thus, $f^{\prime}\left(\left\|x-x^{*}\right\|\right)<0$ which, together (5) and $\mathbf{h} \mathbf{1}$, taking into account (12), imply that for all $x \in B\left(x^{*}, r\right)$

$$
\left\|{\widehat{F^{\prime}\left(x^{*}\right)}}^{-1}\right\|\left\|\widehat{F^{\prime}(x)}-\widehat{F^{\prime}\left(x^{*}\right)}\right\| \leq\left\|{\widehat{F^{\prime}\left(x^{*}\right)}}^{-1}\right\|\left\|F^{\prime}(x)-F^{\prime}\left(x^{*}\right)\right\| \leq f^{\prime}\left(\left\|x-x^{*}\right\|\right)-f^{\prime}(0)<1 .
$$

Thus, by Banach's lemma, we conclude that ${\widehat{F^{\prime}(x)}}^{-1}$ exists. Moreover by above inequality,

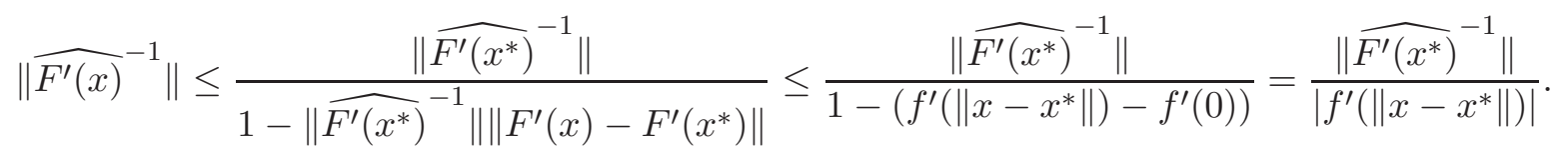

The last result follows by noting that $r=\min \{\kappa, \nu\}$. On the other hand, using (13) we have

$$
\left\|\widehat{F^{\prime}(x)}-\widehat{F^{\prime}\left(x^{*}\right)}\right\| \leq \frac{1}{\| \widehat{F^{\prime}\left(x^{*}\right)}-1} .
$$

Take $y \in H$. Then, it follows by above inequality that

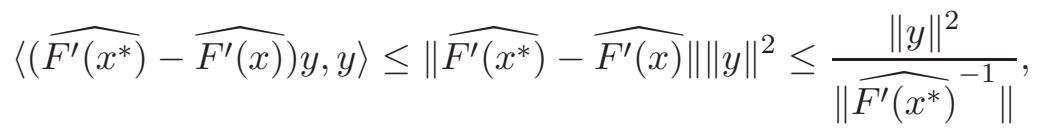

which implies, after of simple manipulations that

$$
\left.\left\langle\widehat{F^{\prime}\left(x^{*}\right)} y, y\right\rangle-\frac{\|y\|^{2}}{\left\|{\widehat{F^{\prime}\left(x^{*}\right)}}^{-1}\right\|} \leq \widehat{F^{\prime}(x)} y, y\right\rangle
$$

Since $\widehat{F^{\prime}\left(x^{*}\right)}$ is a positive operator and ${\widehat{F^{\prime}\left(x^{*}\right)}}^{-1}$ exists by assumption, we obtain by Lemma 2 that

$$
\left\langle\widehat{F^{\prime}\left(x^{*}\right)} y, y\right\rangle \geq \frac{\|y\|^{2}}{\| \widehat{F}^{\prime}\left(x^{*}\right)}-1 \|
$$

Therefore, combining the two last inequalities we conclude that $\left.\widehat{\left\langle F^{\prime}(x)\right.} y, y\right\rangle \geq 0$, i.e., $\widehat{F^{\prime}(x)}$ is a positive operator.

Lemma 8 shows that $\widehat{F^{\prime}(x)}$ is a positive operator and ${\widehat{F^{\prime}(x)}}^{-1}$ exists, thus by Lemma 2 we have that for any $y \in H$

$$
\left.\widehat{\left\langle F^{\prime}(x)\right.} y, y\right\rangle \geq \frac{\|y\|^{2}}{\left\|{\widehat{F^{\prime}(x)}}^{-1}\right\|} .
$$

Note that $\left.\widehat{\left\langle F^{\prime}(x)\right.} y, y\right\rangle=\left\langle F^{\prime}(x) y, y\right\rangle$, thus by the second part of Lemma 8 and $h_{2}$ we conclude that $F^{\prime}(x)$ satisfies (8) and consequently, the Newton iteration mapping is well-defined. Let us call $N_{F+T}$, the Newton iteration mapping for $F+T$ in that region, namely, $N_{F+T}: B\left(x^{*}, r\right) \rightarrow H$ is defined by

$$
0 \in F(x)+F^{\prime}(x)\left(N_{F+T}(x)-x\right)+T\left(N_{F+T}(x)\right), \quad \forall x \in B\left(x^{*}, r\right) .
$$

Therefore, one can apply a single Newton iteration on any $x \in B\left(x^{*}, r\right)$ to obtain $N_{F+T}(x)$ which may not belong to $B\left(x^{*}, r\right)$, or even may not belong to the domain of $F$. Thus, this is enough to guarantee the well-definedness of only one iteration of Newton's method. To ensure that Newtonian iterations may be repeated indefinitely, we need an additional result. 
Lemma 9. Take $0<t<r$. If $\left\|x-x^{*}\right\| \leq t$ then $\left\|N_{F+T}(x)-x^{*}\right\| \leq\left[\left|n_{f}(t)\right| / t^{2}\right]\left\|x-x^{*}\right\|^{2}$. As a consequence, $N_{F+T}\left(B\left[x^{*}, t\right]\right) \subset B\left[x^{*},\left|n_{f}(t)\right|\right]$. Moreover, $N_{F+T}\left(B\left(x^{*}, r\right)\right) \subset B\left(x^{*}, r\right)$.

Proof. Since $0 \in F\left(x^{*}\right)+T\left(x^{*}\right)$ we have $x^{*}=N_{F+T}\left(x^{*}\right)$. Thus, the inequality of the lemma is trivial for $x=x^{*}$. Now, assume that $0<\left\|x-x^{*}\right\| \leq t$. Let $y=N_{F+T}(x)$. By (15) we have $0 \in F(x)+F^{\prime}(x)(y-x)+T(y)$ for all $x \in B\left(x^{*}, r\right)$. As $T$ is a maximal monotone, it follows that

$$
\left\langle F(x)-F\left(x^{*}\right)+F^{\prime}(x)\left(x^{*}-x\right)+F^{\prime}(x)\left(y-x^{*}\right), x^{*}-y\right\rangle \geq 0
$$

which implies that

$$
\left\langle F(x)-F\left(x^{*}\right)+F^{\prime}(x)\left(x^{*}-x\right), x^{*}-y\right\rangle \geq\left\langle F^{\prime}(x)\left(x^{*}-y\right), x^{*}-y\right\rangle .
$$

Since, by Lemma $8, \widehat{F^{\prime}(x)}$ is a positive operator and ${\widehat{F^{\prime}(x)}}^{-1}$ exists, we obtain from Lemma 2 that

$$
\left.\frac{\left\|x^{*}-y\right\|^{2}}{\| \widehat{F}^{\prime}(x)}-1 \widehat{\left\langle F^{\prime}(x)\right.}\left(x^{*}-y\right), x^{*}-y\right\rangle \text {. }
$$

Note that $\left.\widehat{\left\langle F^{\prime}(x)\right.}\left(x^{*}-y\right), x^{*}-y\right\rangle=\left\langle F^{\prime}(x)\left(x^{*}-y\right), x^{*}-y\right\rangle$, this together with (17) and (16) yields that

$$
\left\|x^{*}-y\right\|^{2} \leq\left\|{\widehat{F^{\prime}(x)}}^{-1}\right\|\left\langle F^{\prime}(x)\left(x^{*}-y\right), x^{*}-y\right\rangle \leq\left\|{\widehat{F^{\prime}(x)}}^{-1}\right\|\left\langle F(x)-F\left(x^{*}\right)+F^{\prime}(x)\left(x^{*}-x\right), x^{*}-y\right\rangle .
$$

Hence, after simple manipulations, above inequality becomes

$$
\left\|x^{*}-y\right\| \leq\left\|{\widehat{F^{\prime}(x)}}^{-1}\right\|\left\|F(x)-F\left(x^{*}\right)+F^{\prime}(x)\left(x^{*}-x\right)\right\| .
$$

Using (11), second part in Lemma 8 and Lemma 7 in (18) we obtain that

$$
\left\|x^{*}-y\right\| \leq \frac{\left\|{\widehat{F^{\prime}\left(x^{*}\right)}}^{-1}\right\|}{\left|f^{\prime}\left(\left\|x-x^{*}\right\|\right)\right|}\left\|E_{F}\left(x, x^{*}\right)\right\| \leq \frac{e_{f}\left(\left\|x-x^{*}\right\|, 0\right)}{\left|f^{\prime}\left(\left\|x-x^{*}\right\|\right)\right|} .
$$

On the other hand, taking into account that $f(0)=0$, the definitions of $e_{f}$ and $n_{f}$ imply that

$$
\frac{e_{f}\left(\left\|x-x^{*}\right\|, 0\right)}{\left|f^{\prime}\left(\left\|x-x^{*}\right\|\right)\right|}=-n_{f}\left(\left\|x-x^{*}\right\|\right)=\left|n_{f}\left(\left\|x-x^{*}\right\|\right)\right|
$$

As $\left\|x-x^{*}\right\| \leq t$, the first part of Proposition 5 gives $\left|n_{f}\left(\left\|x-x^{*}\right\|\right)\right| /\left\|x-x^{*}\right\|^{2} \leq\left|n_{f}(t)\right| / t^{2}$, thus the last inequality becomes

$$
\frac{e_{f}\left(\left\|x-x^{*}\right\|, 0\right)}{\left|f^{\prime}\left(\left\|x-x^{*}\right\|\right)\right|} \leq\left|n_{f}(t)\right| / t^{2}\left\|x-x^{*}\right\|^{2}
$$

Hence, the desired inequality follows by combining (19) and the latter equation.

For proving second part of the lemma, take $x \in B\left[x^{*}, t\right]$. Since $\left\|x-x^{*}\right\| \leq t$, first part of the lemma implies that $\left\|N_{F+T}(x)-x^{*}\right\| \leq\left|n_{f}(t)\right|$, and the first inclusion follows. Due to $r \leq \rho$, second part of Proposition 5 implies that $\left|n_{f}(t)\right| \leq t$. Thus, the last inclusion is an immediate consequence of the first one.

In the next result we obtain the uniqueness of the solution in the neighborhood $B[\bar{x}, \sigma]$. 
Lemma 10. Take $t \in(0, r)$ and suppose that $F^{\prime}\left(x^{*}\right)$ is a positive operator and ${\widehat{F^{\prime}\left(x^{*}\right)}}^{-1}$ exists. If $f(t)<0$, i.e., 0 is the unique zero of $f$ in $[0, t]$, then $x^{*}$ is the unique solution of (11) in $B\left[x^{*}, t\right]$. As consequence, $x^{*}$ is the unique solution of (11) in $B\left[x^{*}, \bar{\sigma}\right]$.

Proof. Assume that $y \in B\left[x^{*}, t\right]$ and $0 \in F(y)+T(y)$. Then, as $T$ is a maximal monotone and $0 \in F\left(x^{*}\right)+T\left(x^{*}\right)$ we obtain that

$$
\left\langle F(y)-F\left(x^{*}\right), x^{*}-y\right\rangle \geq 0
$$

which implies that $\left\langle F(y)-F\left(x^{*}\right)-F^{\prime}\left(x^{*}\right)\left(y-x^{*}\right)+F^{\prime}\left(x^{*}\right)\left(y-x^{*}\right), x^{*}-y\right\rangle \geq 0$ and thus

$$
\left\langle F(y)-F\left(x^{*}\right)-F^{\prime}\left(x^{*}\right)\left(y-x^{*}\right), x^{*}-y\right\rangle \geq\left\langle F^{\prime}\left(x^{*}\right)\left(x^{*}-y\right),\left(x^{*}-y\right)\right\rangle .
$$

Since $F^{\prime}\left(x^{*}\right)$ is a positive operator and ${\widehat{F^{\prime}\left(x^{*}\right)}}^{-1}$ exists, we can apply Lemma 2 to obtain that

$$
\left\langle F^{\prime}\left(x^{*}\right)\left(x^{*}-y\right),\left(x^{*}-y\right)\right\rangle=\left\langle\widehat{F^{\prime}\left(x^{*}\right)}\left(x^{*}-y\right),\left(x^{*}-y\right)\right\rangle \geq \frac{\left\|x^{*}-y\right\|^{2}}{\left\|{\widehat{F}\left(x^{*}\right)}^{-1}\right\|} .
$$

On the other hand

$$
F(y)-F\left(x^{*}\right)-F^{\prime}\left(x^{*}\right)\left(y-x^{*}\right)=\int_{0}^{1}\left[F^{\prime}\left(x^{*}+t\left(y-x^{*}\right)\right)-F^{\prime}\left(x^{*}\right)\right]\left(y-x^{*}\right) d t .
$$

Combining above equality with (21) and (20), yields that

$$
\left\|y-x^{*}\right\| \leq \int_{0}^{1}\left\|{\widehat{F^{\prime}\left(x^{*}\right)}}^{-1}\right\|\left\|F^{\prime}\left(x^{*}+t\left(y-x^{*}\right)\right)-F^{\prime}\left(x^{*}\right)\right\|\left\|\left(y-x^{*}\right)\right\| d t .
$$

Using (5) with $x=x^{*}+t\left(y-x^{*}\right)$ and $\tau=0$ it is easy to conclude from the last equality that

$$
\left\|y-x^{*}\right\| \leq \int_{0}^{1}\left[f^{\prime}\left(t\left\|y-x^{*}\right\|\right)-f^{\prime}(0)\right]\left\|y-x^{*}\right\| d t=f\left(\left\|y-x^{*}\right\|\right)-f(0)-f^{\prime}(0)\left\|y-x^{*}\right\| .
$$

Taking into account that $f(0)=0$ and $f^{\prime}(0)=-1$ the latter inequality becomes

$$
f\left(\left\|y-x^{*}\right\|\right) \geq 0 .
$$

Since $f$ is strictly convex and $f(t)<0$, we will have $f<0$ in $(0, t]$, i.e., 0 is the unique zero of $f$ in $[0, t]$ and hence, the above inequality implies that $\left\|y-x^{*}\right\|=0$, i.e., $y=x^{*}$. Thus, $x^{*}$ is the unique zero of $F+T \ni 0$ in $B\left[x^{*}, t\right]$. The second part follows from the definition of $\sigma$.

In the next result we will obtain the the optimal convergence radius, which has its proof similar to the proof of Lemma 2.15 of [9].

Lemma 11. If $f(\rho) /\left(\rho f^{\prime}(\rho)\right)-1=1$ and $\rho<\kappa$, then $r=\rho$ is the optimal convergence radius.

\subsection{Proof of Theorem 4}

Firstly, it is easy to see that the inclusion in (6) together (15) imply that the sequence $\left\{x_{k}\right\}$ satisfies

$$
0 \in F\left(x_{k}\right)+F^{\prime}\left(x_{k}\right)\left(N_{F+T}\left(x_{k}\right)-x_{k}\right)+T\left(N_{F+T}\left(x_{k}\right)\right), \quad k=0,1, \ldots
$$


Proof. That $\left\{t_{k}\right\}$ is well defined, is strictly decreasing and is contained in $(0, \rho)$ follows from Corollary 6. Moreover, from this same corollary, we conclude that $\left\{t_{k+1} / t_{k}^{2}\right\}$ is strictly decreasing, $\left\{t_{k}\right\}$ converges to 0 and $t_{k+1} / t_{k}^{2} \leq\left[f^{\prime \prime}\left(t_{0}\right) /\left(2\left|f^{\prime}\left(t_{0}\right)\right|\right)\right]$, for $k=0,1, \ldots$

As $x_{0} \in B\left(x^{*}, r\right)$, and $r \leq \nu$, we conclude by combining (22) and inclusion $N_{F+T}\left(B\left(x^{*}, r\right)\right) \subset$ $B\left(x^{*}, r\right)$ in second part of Lemma 9 that $\left\{x_{k}\right\}$ is well defined and remains in $B\left(x^{*}, r\right)$. On the other hand, since $0<\left\|x_{k}-x^{*}\right\|<r \leq \rho$, for $k=0,1, \ldots$, we obtain from (22), Lemma 9 and second part of Proposition 5 that

$$
\left\|x_{k+1}-x^{*}\right\| \leq\left|n_{f}\left(\left\|x_{k}-x^{*}\right\|\right)\right|<\left\|x_{k}-x^{*}\right\|, \quad k=0,1, \ldots
$$

Thence, $\left\{\left\|x_{k}-x^{*}\right\|\right\}$ is strictly decreasing and convergent. Let $b=\lim _{k \rightarrow \infty}\left\|x_{k}-x^{*}\right\|$. Because $\left\{\left\|x_{k}-x^{*}\right\|\right\}$ rest in $(0, \rho)$ and it is strictly decreasing we have $0 \leq b<\rho$. Then, by continuity of $n_{f}$ and (23) imply $0 \leq b=\left|n_{f}(b)\right|$, and from second part of Proposition 5 we have $b=0$. Therefore, we conclude that $\left\{x_{k}\right\}$ converges to $x^{*}$. Due to $t_{0}=\left\|x^{*}-x_{0}\right\|$, definition of $\left\{t_{k}\right\}$ in (10) implies that $t_{k+1}=\left|n_{f}\left(t_{k}\right)\right|$, hence (22) and Lemma 9 imply that

$$
\left\|x_{k+1}-x^{*}\right\|=\left\|N_{F+T}\left(x_{k}\right)-x^{*}\right\| \leq\left|n_{f}\left(t_{k}\right)\right|, \quad k=0,1, \ldots
$$

Then, the first inequality in (77) follows from last inequality, first part of Lemma 9 and the definition of $\left\{t_{k}\right\}$ in (10). Finally, the uniqueness follows from Lemma 10 and the last statement in the theorem follows from Lemma 11.

\section{Some special cases}

In this section, we will present some special cases of Theorem 4. When $F \equiv\{0\}$ and $f^{\prime}$ satisfies a Lipschitz-type condition, we will obtain a particular instance of Theorem 4 , which retrieves the classical convergence theorem on Newton's method under the Lipschitz condition; see [16, 19]. A version of Smale's theorem on Newton's method for analytical functions is obtained in Theorem 13 .

\subsection{Under Lipschitz-type condition}

In this section, we will present a version of classical convergence theorem for Newton's method under Lipschitz-type condition for generalized equations. The classical version for $F \equiv\{0\}$ have appeared in Rall [16] and Traub and Wozniakowski [19].

Theorem 12. Let $H$ be a Hilbert space, $\Omega$ be an open nonempty subset of $H, F: \Omega \rightarrow H$ be continuous with Fréchet derivative $F^{\prime}$ continuous, $T: H \rightrightarrows H$ be a set-valued operator and $x^{*} \in \Omega$. Suppose that $0 \in F\left(x^{*}\right)+T\left(x^{*}\right), F^{\prime}\left(x^{*}\right)$ is a positive operator and ${\widehat{F^{\prime}\left(x^{*}\right)}}^{-1}$ exists and, there exists a constant $K>0$ such that

$$
\left\|{\widehat{F^{\prime}\left(x^{*}\right)}}^{-1}\right\|\left\|f^{\prime}(x)-f^{\prime}(y)\right\| \leq K\|x-y\|, \quad x, y \in \Omega .
$$

Let $r:=\min \{\kappa, 2 /(3 K)\}$, where $\kappa:=\sup \left\{t>0: B\left(x^{*}, t\right) \subset \Omega\right\}$. Then, the sequences with starting point $x_{0} \in B\left(x^{*}, r\right) /\left\{x^{*}\right\}$ and $t_{0}=\left\|x^{*}-x_{0}\right\|$, respectively,

$$
F\left(x_{k}\right)+F^{\prime}\left(x_{k}\right)\left(x_{k+1}-x_{k}\right)+T\left(x_{k+1}\right) \ni 0, \quad t_{k+1}=\left((K / 2) t_{k}^{2}\right) /\left(1-K t_{k}\right), \quad k=0,1, \ldots,
$$

are well defined, $\left\{t_{k}\right\}$ is strictly decreasing, is contained in $(0, r)$ and converges to $0,\left\{x_{k}\right\}$ is contained in $B\left(x^{*}, r\right)$ and converges to the point $x^{*}$ which is the unique solution of $F(x)+T(x) \ni 0$ in 
$B\left(x^{*}, \bar{\sigma}\right)$, where $\bar{\sigma}=\min \{r, 2 / K\}$. Moreover, $\left\{t_{k+1} / t_{k}^{2}\right\}$ is strictly decreasing, $t_{k+1} / t_{k}^{2}<1 /[2 / K-$ $\left.2\left\|x^{*}-x_{0}\right\|\right]$ and

$$
\left\|x^{*}-x_{k+1}\right\| \leq \frac{K}{2} \frac{1}{1-K t_{k}}\left\|x_{k}-x^{*}\right\|^{2} \leq \frac{K}{2} \frac{1}{1-K\left\|x_{0}-x^{*}\right\|}\left\|x_{k}-x^{*}\right\|^{2}, \quad k=0,1, \ldots
$$

If, additionally, $2 /(3 K)<\kappa$, then $r=2 /(3 K)$ is the best possible convergence radius.

Proof. Using condition in (24), we can immediately prove that $F, x^{*}$ and $f:[0, \kappa) \rightarrow \mathbb{R}$, defined by $f(t)=K t^{2} / 2-t$, satisfy the inequality (5) and the conditions $\mathbf{h} \mathbf{1}$ and $\mathbf{h} \mathbf{2}$ in Theorem 4. In this case, it is easy to see that $\rho$ and $\nu$, as defined in Theorem 4 , satisfy $\rho=2 /(3 K) \leq \nu=1 / K$ and, as a consequence, $r:=\min \{\kappa, 2 /(3 K)\}$. Moreover, $f(\rho) /\left(\rho f^{\prime}(\rho)\right)-1=1, f(0)=f(2 / K)=0$ and $f(t)<0$ for all $t \in(0,2 / K)$. Also, the sequence $\left\{t_{k}\right\}$ in Theorem 4 is given by (25) and

$$
t_{k+1} / t_{k}^{2}=\frac{K}{2} \frac{1}{1-K t_{k}}<\frac{K}{2} \frac{1}{1-K\left\|x_{0}-x^{*}\right\|}, \quad k=0,1, \ldots
$$

Therefore, the result follows by invoking Theorem 4 .

Remark 3. The above result contain, as particular instance, several theorem on Newton's method; see, for example, Rall [16], Traub and Wozniakowski [19] and Daniel [3].

Remark 4. Since $\left\|x^{*}-x_{0}\right\| \leq 2 /(3 K)$, the last inequality in (26) implies that $\left\|x^{*}-x_{k+1}\right\| \leq$ $3 K / 2\left\|x^{*}-x_{k}\right\|^{2}$ for $k=0,1, \ldots$ Then, we conclude that

$$
\left\|x^{*}-x_{k}\right\| \leq \frac{2}{3 K}\left(\frac{3 K}{2}\left\|x^{*}-x_{0}\right\|\right)^{2^{k}}, \quad k=0,1, \ldots
$$

\subsection{Under Smale's-type condition}

In this section, we will present a version of classical convergence theorem for Newton's method under Smale's-type condition for generalized equations. The classical version has appeared in corollary of Proposition 3 pp. 195 of Smale [18], see also Proposition 1 pp. 157 and Remark 1 pp. 158 of Blum, Cucker, Shub, and Smale [1]; see also [9].

Theorem 13. Let $H$ be a Hilbert space, $\Omega$ be an open nonempty subset of $H, F: \Omega \rightarrow H$ be an analytic function, $T: H \rightrightarrows H$ be a set-valued operator and $x^{*} \in \Omega$. Suppose that $0 \in F\left(x^{*}\right)+T\left(x^{*}\right)$, $F^{\prime}\left(x^{*}\right)$ is a positive operator and ${\widehat{F^{\prime}\left(x^{*}\right)}}^{-1}$ exists. Suppose that

$$
\gamma:=\left\|{\widehat{F^{\prime}\left(x^{*}\right)}}^{-1}\right\| \sup _{n>1}\left\|\frac{F^{(n)}\left(x^{*}\right)}{n !}\right\|^{1 /(n-1)}<+\infty .
$$

Let $r=\min \{\kappa,(5-\sqrt{17}) /(4 \gamma)\}$, where $\kappa:=\sup \left\{t>0: B\left(x^{*}, t\right) \subset \Omega\right\}$. Then, the sequences with starting point $x_{0} \in B\left(x^{*}, r\right) /\left\{x^{*}\right\}$ and $t_{0}=\left\|x^{*}-x_{0}\right\|$, respectively

$$
0 \in F\left(x_{k}\right)+F^{\prime}\left(x_{k}\right)\left(x_{k+1}-x_{k}\right)+T\left(x_{k+1}\right), \quad t_{k+1}=\gamma t_{k}^{2} /\left[2\left(1-\gamma t_{k}\right)^{2}-1\right], \quad k=0,1, \ldots,
$$

are well defined, $\left\{t_{k}\right\}$ is strictly decreasing, contained in $(0, r)$ and converges to 0 , and $\left\{x_{k}\right\}$ is contained in $B\left(x^{*}, r\right)$ and converges to the point $x^{*}$ which is the unique solution of $F(x)+T(x) \ni$ 0 in $B\left(x^{*}, \bar{\sigma}\right)$, where $\bar{\sigma}=\min \{r, 1 /(2 \gamma)\}$. Moreover, $\left\{t_{k+1} / t_{k}^{2}\right\}$ is strictly decreasing, $t_{k+1} / t_{k}^{2}<$ $\gamma /\left[2\left(1-\gamma\left\|x_{0}-x^{*}\right\|\right)^{2}-1\right]$, for $k=0,1, \ldots$ and

$$
\left\|x_{k+1}-x^{*}\right\| \leq \frac{\gamma}{2\left(1-\gamma t_{k}\right)^{2}-1}\left\|x_{k}-x^{*}\right\|^{2} \leq \frac{\gamma}{2\left(1-\gamma\left\|x_{0}-x^{*}\right\|\right)^{2}-1}\left\|x_{k}-x^{*}\right\|^{2}, \quad k=0,1, \ldots
$$

If, additionally, $(5-\sqrt{17}) /(4 \gamma)<\kappa$, then $r=(5-\sqrt{17}) /(4 \gamma)$ is the best possible convergence radius. 
Before proving above theorem we need of two results. The next results gives a condition that is easier to check than condition (5), whenever the functions under consideration are twice continuously differentiable, and its proof follows the same path of Lemma 21 of [10].

Lemma 14. Let $\Omega \subset H$ be an open set, and let $F: \Omega \rightarrow H$ be an analytic function. Suppose that $x^{*} \in \Omega$ and $B\left(x^{*}, 1 / \gamma\right) \subset \Omega$, where $\gamma$ is defined in (27). Then for all $x \in B\left(x^{*}, 1 / \gamma\right)$, it holds that $\left\|F^{\prime \prime}(x)\right\| \leq 2 \gamma /\left(1-\gamma\left\|x-x^{*}\right\|\right)^{3}$.

The next result gives a relationship between the second derivatives $F^{\prime \prime}$ and $f^{\prime \prime}$, which allow us to show that $F$ and $f$ satisfy (5), and its proof is similar to Lemma 22 of [10].

Lemma 15. Let $H$ be a Hilbert space, $\Omega \subseteq H$ be an open set, $F: \Omega \rightarrow H$ be twice continuously differentiable. Let $x^{*} \in \Omega, R>0$ and $\kappa=\sup \left\{t \in[0, R): B\left(x^{*}, t\right) \subset \Omega\right\}$. Let $f:[0, R) \rightarrow \mathbb{R}$ be twice continuously differentiable such that $\left\|{\widehat{F^{\prime}\left(x^{*}\right)}}^{-1}\right\|\left\|F^{\prime \prime}(x)\right\| \leqslant f^{\prime \prime}\left(\left\|x-x^{*}\right\|\right)$, for all $x \in B\left(x^{*}, \kappa\right)$, then $F$ and $f$ satisfy (5).

[Proof of Theorem 13]. Consider $f:[0,1 / \gamma) \rightarrow \mathbb{R}$ defined by $f(t)=t /(1-\gamma t)-2 t$. Note that $f$ is analytic and $f(0)=0, f^{\prime}(t)=1 /(1-\gamma t)^{2}-2, f^{\prime}(0)=-1, f^{\prime \prime}(t)=2 \gamma /(1-\gamma t)^{3}$. It follows from the last equalities that $f$ satisfies $\mathbf{h 1}$ and $\mathbf{h 2}$. Combining Lemma 15 with Lemma 14, we conclude that $F$ and $f$ satisfy (5). The constants, $\nu, \rho$ and $r$, as defined in Theorem 4 , satisfy

$$
\rho=\frac{5-\sqrt{17}}{4 \gamma}<\nu=\frac{\sqrt{2}-1}{\sqrt{2} \gamma}<\frac{1}{\gamma}, \quad r=\min \left\{\kappa, \frac{5-\sqrt{17}}{4 \gamma}\right\} .
$$

Moreover, $f(\rho) /\left(\rho f^{\prime}(\rho)\right)-1=1$ and $f(0)=f(1 /(2 \gamma))=0$ and $f(t)<0$ for $t \in(0,1 /(2 \gamma))$. Also, $\left\{t_{k}\right\}$ satisfy

$$
t_{k+1} / t_{k}^{2}=\frac{\gamma}{2\left(1-\gamma t_{k}\right)^{2}-1}<\frac{\gamma}{2\left(1-\gamma\left\|x_{0}-x^{*}\right\|\right)^{2}-1}, \quad k=0,1, \ldots
$$

Therefore, the result follows by applying the Theorem 4 .

\section{References}

[1] L. Blum, F. Cucker, M. Shub, and S. Smale. Complexity and real computation. Springer-Verlag, New York, 1998. With a foreword by Richard M. Karp.

[2] D. C. Chang, J. Wang, J. C. Yao Newtons method for variational inequality problems: Smales point estimate theory under the $\gamma$-condition. Applicable Analysis, 94(1):44-55, 2015.

[3] J. W. Daniel. Newton's method for nonlinear inequalities. Numer. Math., 21:381-387, 1973.

[4] S. P. Dokov, A. L. Dontchev. Robinson's strong regularity implies robust local convergence of Newton's method Optimal control (Gainesville, FL, 1997), vol. 15 of Appl. Optim., pages 116-129 Kluwer Acad. Publ., Dordrecht, 1998.

[5] A. L. Dontchev. Local analysis of a Newton-type method based on partial linearization. In The mathematics of numerical analysis (Park City, UT,1995). Amer. Math. Soc., Providence, RI, 1996.

[6] A. L. Dontchev. Local convergence of the Newton method for generalized equations $C$. $R$. Acad. Sci. Paris Sér. I Math., 322(4):327-331, 1996. 
[7] A. L. Dontchev and R. T. Rockafellar. Implicit functions and solution mappings. Springer Monographs in Mathematics. Springer, Dordrecht, 2009. A view from variational analysis.

[8] A. L. Dontchev and R. T. Rockafellar. Newton's method for generalized equations: a sequential implicit function theorem. Math. Program., 123(1, Ser.B):139-159,2010.

[9] O. Ferreira. Local convergence of Newton's method in Banach space from the viewpoint of the majorant principle. IMA J. Numer. Anal., 29(3)746-759, 2009.

[10] O. P. Ferreira, M. L. N. Gonçalves, and P. R. Oliveira. Local convergence analysis of the Gauss-Newton method under a majorant condition. J. Complexity., 27(1):111-125, 2011.

[11] O. P. Ferreira, G. N. Silva. Inexact Newton's method to nonlinear functions with values in a cone. arXiv:1510.01947, 2015.

[12] O. P. Ferreira and B. F. Svaiter. Kantorovich's majorants principle for Newton's method. Comput. Optim. Appl., 42(2):213-229, 2009.

[13] G. N. Silva. Kantorovichs theorem on Newtons method for solving generalized equations under the majorant condition. Applied Mathematics and Computation, 286:178-188, 2016.

[14] M. Josephy. Newton's Method for Generalized Equations and the PIES Energy Model. University of Wisconsin-Madison, 1979.

[15] A. Pietrus and C. Jean-Alexis. Newton-secant method for functions with values in a cone. Serdica Math. J., 39(3-4):271-286, 2013.

[16] L. B. Rall. A Note on the Convergence of Newton's Method. SIAM Journal on Numerical Analysis , 11(1):pp. 34-36, 1974.

[17] S. M. Robinson. Extension of Newton's method to nonlinear functions with values in a cone. Numer. Math., 19:341-347, 1972.

[18] S. Smale. Newtons Method Estimates from Data at One Point. The Merging of Disciplines: New Directions in Pure, Applied, and Computational Mathematics Springer New York, pages 185-196, 1986.

[19] J. F. Traub, H. Woźniakowski. Convergence and complexity of Newton iteration for operator equations. J. Assoc. Comput. Mach., 26(2):250-258, 1979.

[20] L. U. Uko. Generalized equations and the generalized Newton method Math. Programming, 73(3, Ser. A):251-268, 1996.

[21] L. U. Uko, I. K. Argyros. Generalized equations, variational inequalities and a weak Kantorovich theorem Numerical Algorithms, 52(3):321-333, 2009.

[22] J. Wang. Convergence ball of Newton's method for generalized equation and uniqueness of the solution J. Nonlinear Convex Anal., 16(9):1847-1859, 2015.

[23] X. Wang. Convergence of Newton's method and inverse function theorem in Banach space Math. Comp., 68(225):169-186, 1999.

[24] P. P. Zabrejko, D. F. Nguen The majorant method in the theory of Newton-Kantorovich approximations and the Pták error estimates Numer. Funct. Anal. Optim., 9(5-6):671-684, 1987. 